\title{
No difference in anterior tibial translation with and without posterior cruciate ligament in less invasive total knee replacement
}

\author{
Bernhard Christen • Michal Neukamp • \\ Emin Aghayev
}

Received: 1 December 2010/Accepted: 19 May 2011/Published online: 1 June 2011

(C) The Author(s) 2011. This article is published with open access at Springerlink.com

\begin{abstract}
Purpose The relative advantages of cruciate retaining or cruciate resecting total knee replacement are still controversial. If the posterior cruciate ligament (PCL) is preserved, it should be properly balanced. In a previous study, it was demonstrated that increasing the flexion gap leads to an anterior translation of the tibia relative to the femur. Based on these results, we hypothesized that cutting the PCL increases the flexion gap and lessens anterior tibial translation.

Methods The amount of anterior tibial translation versus distraction force in the flexion gap was measured in 88 total knee replacements with a less invasive midvastus approach using a custom-made tensioner. Measurements were performed with intact and resected PCL.

Results The difference in tibial translation with and without PCL is not significant. A 1-mm increase in the flexion gap led to an average anterior translation of $0.6 \mathrm{~mm}$ with intact PCL and $0.4 \mathrm{~mm}$ with cut PCL, which is less than that reported in a previous study.

Conclusions The results have not confirmed our initial hypothesis. The reasons for this may be other soft tissue structures that prevent anterior tibial translation, such as the collateral ligaments, and/or the extensor apparatus. Moreover, the knee flexion angle for the used specific implant may play a role.
\end{abstract}

B. Christen $(\square)$

Department of Orthopaedic Surgery, Salem Spital,

Bern, Schänzlistrasse 39, 3000 Bern 25, Switzerland

e-mail: bernhard.christen@ ortho-klinik.ch

M. Neukamp · E. Aghayev

Institute for Evaluative Research in Medicine,

University of Bern, Stauffacherstrasse 78,

3014 Bern, Switzerland
Level of evidence Prospective comparative study, Level II.

Keywords Ligament balancing - Posterior cruciate ligament - Total knee replacement $\cdot$ Anterior tibial translation $\cdot$ Flexion gap

\section{Introduction}

The role of the posterior cruciate ligament (PCL) in total knee replacement (TKR) is seen as relevant to the restoration of the natural range of motion (ROM) [1]. Being the strongest ligament in the knee joint, the PCL pulls the femur posteriorly onto the tibia in flexion "roll-back" $[1,13]$ and prevents posterior translation of the tibia, respectively, resists anterior femoral translation [8] in motion and, thus, may influence the femorotibial contact point.

Two general approaches exist regarding the role of the PCL in TKR. Depending upon operative technique and the implant, some surgeons resect the ligament, while others prefer keeping the PCL intact for further stabilization of the new joint. Although discussion of this issue is relatively old, a consensus has not yet been reached [13].

Good mid- and long-term results are described for both cruciate ligament retaining total knee replacements (CR TKR) and posterior stabilized total knee replacements (PS TKR) $[16,25]$. Several studies including also meta-analyses comparing CR TKR and PS TKR were performed, but no clear significant benefits of one knee design over the other was demonstrated $[13,14,17]$. Instability due to insufficiency of the PCL after CR TKR is also a welldocumented problem $[19,22,27]$. The difficulties with and importance of PCL balancing in TKR have been demonstrated in several studies $[9,18,23,26]$. Ligament balancing became important in TKR as a correlation was 
assumed between laxity in the sagittal plane, postoperative ROM and knee function [12]. However, subsequent investigations have not confirmed the correlation [24, 29]. Dejour et al. [4] compared 118 PS TKR with 138 CR TKR and found that the latter had significantly more clinical and radiological laxity [4]. Analyses of kinematics in TKR have found paradoxical anterior sliding and opposite rotational axial patterns during a deep knee bend in patients with CR TKR, whereas patients with PS TKR showed less variability $[5,6]$. The authors also have documented significantly higher ROM under weight-bearing conditions in patients after PS TKR $\left(113^{\circ}\right)$ compared with CR TKR $\left(103^{\circ}\right)$. Overall, limited scientific evidence informs the choice between a PS and a CR TKR [14].

A quantitative analysis of the relationship between the size of the flexion gap and the anterior translation of the tibia in flexion in CR TKR was recently performed [2]. The study showed that with an increasing flexion gap, the tibia tends to translate anteriorly because of the rising tension in the PCL and posterior capsule. Similarly, Heesterbeek et al. [10] found significant correlation between flexion gap height and anterior tibial translation in their quantitative analysis in 50 navigated CR TKR patients. The goal of this study was to reproduce the results with a less invasive surgical approach and a PS TKR, allowing assessment of the relationship between flexion gap and tibial translation both with and without the PCL. We hypothesized that the tibial translation is more pronounced with an intact PCL than after its resection.

\section{Materials and methods}

In a consecutive series of 114 knees in 100 patients, a bicruciate stabilized total knee replacement (Journey, Smith \& Nephew, Memphis, USA) was performed by a single surgeon between December 2006 and March 2009. Thirteen knees had to be excluded due to previous knee surgery, a missing or torn PCL or an incomplete data set. A further 13 fixed valgus knees were not included in this study as they needed a lateral approach including an osteotomy of the tibial tubercle. The remaining 88 knees (38 were right and 50 were left knees) in 87 patients with osteoarthritis were included in the study. Sixty patients were women with mean age 69 years, and 27 were men with mean age 67 years. There were 65 varus (73\%), 15 neutral (17\%) and 8 slight and redressable valgus (9\%) alignments of the preoperative leg axis in 88 knees.

Journey bicruciate stabilized knee system (BCS)

To understand some procedural details described below, certain features of the Journey knee prosthesis (Smith \&
Nephew, Memphis, USA) have to be mentioned. The Journey BCS demands a resection of both cruciate ligaments and tries to reconstruct their function by a post-cam mechanism not only for the PCL but the anterior cam slope to simulate the anterior cruciate ligament (ACL). The desired tibial cut is perpendicular in the coronal plane and has $3^{\circ}$ of posterior slope in the sagittal plane. The femoral component is asymmetrical and has a smaller radius on the lateral compared with the medial condyle. This difference is compensated by an insert, the lateral part of which is thicker and produces a varus tilt of $3^{\circ}$. Furthermore, the anterior and posterior femoral cuts in the coronal plane are not parallel but converging. Whereas the anterior cut aims to be flush on the anterior femoral cortex, the posterior cut is ascending by $15^{\circ}$, which saves bone distally and allows a more accurate coverage of the posterior femoral condyles proximally. The posterior cut influences the knee flexion angle requiring by bringing both tibial and posterior femoral osteotomies parallel to the balance flexion gap. Therefore, the total flexion needed is $108^{\circ}$ resulting from $90^{\circ}$ of flexion, $3^{\circ}$ of posterior tibial slope and $15^{\circ}$ for the ascending osteotomy of the posterior condyles.

\section{Approach and surgical technique}

All 88 knees were accessed by a less invasive midvastus approach. The set-up included a stepless adjustable hydraulic leg holder (knee positioning device 1004.84, Maquet GmbH \& Co KG, Rastatt, Germany) which supported the thigh at the level of the tourniquet and allowed positioning of the knee in any flexion angle desired between 20 and $130^{\circ}$. The foot was still in contact with the operating table up to a knee flexion angle of about $120^{\circ}$. The arthrotomy was performed medial to the patella and distally medial to the patellar ligament. Proximally, the incision was lengthened about $3 \mathrm{~cm}$ into the muscle belly of the vastus medialis obliquus. First, all osteophytes, remnants of the menisci and the anterior cruciate ligament were removed. Tibial osteotomy was then performed using standard instrumentation with extramedullary alignment. The tibial cutting block was attached to the jig and adjusted with a ruler, thus defining varus-valgus alignment, the desired posterior slope of $3^{\circ}$ and the amount of resection of the tibial plateau $(9 \mathrm{~mm}$ below the original medial or $12 \mathrm{~mm}$ below the original lateral joint line). The PCL was left intact by leaving a protecting bone block of the tibial plateau. The extension gap was symmetrically balanced using a double spring tensioner (Mathys Ltd., Bettlach, Switzerland) with a force of 150 newtons (N). In case of a fixed varus deformity, a stepwise release was performed on the concave side. Only when balancing gave a straight leg was the distal femoral osteotomy performed using the standard instrumentation with intramedullary aiming. 

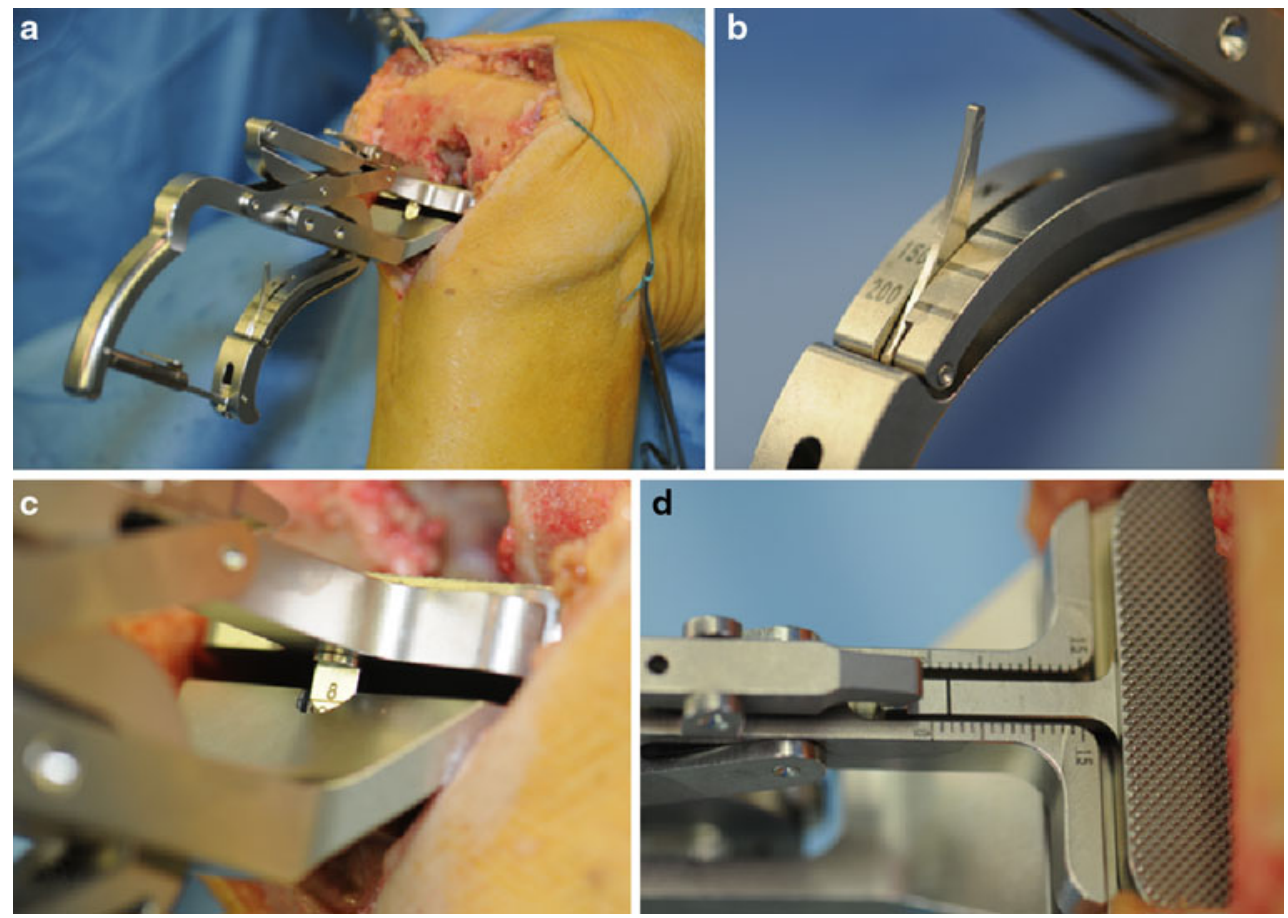

Fig. 1 The figure shows intraoperative views of the tensioner [2] during a placement in the knee joint, $\mathbf{b}$ selection of the tension force, c measurement of flexion gap and $\mathbf{d}$ measurement of tibial translation

The femoral valgus angle was determined preoperatively from long radiographs of the leg and could be reproduced by mounting the appropriate valgus guide to the femoral cutting block.

In the next step, the second femoral cutting block was inserted in $90^{\circ}$ of flexion to define the femoral component size and rotation. A ruler placed on the anterior femoral cortex was used to match the anterior-posterior femoral diameter to the most appropriate femoral component size. In case of intermediate sizes, the correct component could be selected by measuring the medio-lateral femoral diameter. For the rotation, all bony landmarks were respected and then matched with the ligamentous orientation by again using the double spring tensioner with a symmetrical force of $150 \mathrm{~N}$. Then, the femoral cuts were performed with the appropriately sized five-in-one cutting jig. If necessary, remnant posterior osteophytes on the femoral side were removed.

\section{Measurements}

After creating equalized rectangular extension and flexion gaps, the measurements for this study were performed. In all included knees, extensor apparatus was laterally subluxed without everting the patella. The knee was put in flexion that positioned the tibial osteotomy parallel to the posterior femoral cuts in the sagittal plane by the stepless adjustable hydraulic knee positioning device 1004.84
(Maquet $\mathrm{GmbH} \&$ Co KG, Rastatt, Germany) with the patient foot still in contact with the operating table. Then, a custom-made monoblock tensioner was inserted to measure the amount of flexion gap and anterior tibial translation in $\mathrm{mm}$ with forces of 100, 150 and $200 \mathrm{~N}$ (Fig. 1). The spring mechanism and ruler to determine the tension of 100, 150 and $200 \mathrm{~N}$ were the same as in the standard tensioner designed for the Mathys balanSys knee system (Mathys Ltd, Bettlach, Switzerland) (Fig. 1). Each spreader is calibrated in a standardized way; the laser marks are performed individually where the force corresponds to 100 , 150 and $200 \mathrm{~N}$, respectively. The translation in between the tensioner construction is guaranteed by an industrial linear gliding bearing with almost no friction (Fig. 1). Calibration of the rulers for measuring gap size and amount of translation was done by laser marks in polyethylene (PE) thickness increments 8, 10.5, 13 and 15.5 for the size of the flexion gap and in $\mathrm{mm}$ steps for the translation. The accuracy for gap size and translation was $0.5 \mathrm{~mm}$, which was the smallest possible increment. At the moment of measurement, the knee flexion could be fine tuned to exclude any anterior or posterior gaping if the tibial and posterior femoral osteotomies were not perfectly parallel. The thickness of the tensioner was $14 \mathrm{~mm}$. The total flexion gap was therefore the measured gap plus $14 \mathrm{~mm}$. The gap size in $\mathrm{mm}$ was recorded, when the tension was applied, and then, after releasing the locking mechanism of the tensioner, the translation was documented. 
The tensioner was completely released after each applied force. After complete resection of the remaining tibial bone block and PCL, the measurements were repeated in the same way.

Relationship between the gap size and tibial translation (slope)

To identify this relationship, the increasing differences were calculated in the same manner as in the Heesterbeek et al. study [10]. $\Delta$ gap1 and $\Delta$ gap2 were defined as the differences between the flexion gaps at 100 and $150 \mathrm{~N}$, and at 150 and $200 \mathrm{~N}$, respectively. $\Delta$ trans 1 and $\Delta$ trans 2 were defined as the differences in the tibial translation in the same manner. In order to calculate the relation between anterior tibial translation and the gap size, the mathematical slopes were determined.

PCLslope $1=\Delta$ trans $1 \div \Delta$ gap 1

and

PCLslope $2=\Delta \operatorname{trans} 2 \div \Delta$ gap 2

Evaluation and statistical methods

Descriptive assessments of the measurements as well as their stratification by the status of PCL and gender were carried out. The differences between PCL slopes were compared using a Wilcoxon signed-rank test. For the correlation between applied force and flexion gap or translation, the Spearman coefficient was calculated. The level of significance was set to $P \leq 0.05$ throughout the study. All statistical analyses were conducted using SAS 9.2 (SAS Institute Inc, Cary, NC).
Table 1 Flexion gap and anterior tibial translation (mm)

\begin{tabular}{lcll}
\hline Tension force & $\begin{array}{l}\text { PCL intact } \\
\text { Mean (SD) }\end{array}$ & $\begin{array}{l}\text { PCL resected } \\
\text { Mean (SD) }\end{array}$ & $\begin{array}{l}\text { Intact versus } \\
\text { resected PCL }\end{array}$ \\
\hline $\begin{array}{l}\text { Flexion gap } \\
100 \mathrm{~N}\end{array}$ & $6.8(0.5)$ & $7.1(0.9)$ & $P<0.001$ \\
$150 \mathrm{~N}$ & $7.9(1.3)$ & $8.4(1.7)$ & $P<0.001$ \\
$200 \mathrm{~N}$ & $9.2(1.9)$ & $9.5(2.3)$ & $P<0.001$ \\
Anterior tibial translation & & \\
$100 \mathrm{~N}$ & $0.6(0.6)$ & $0.6(0.8)$ & n.s. \\
$150 \mathrm{~N}$ & $1.1(1.0)$ & $1.0(0.8)$ & n.s.* \\
$200 \mathrm{~N}$ & $1.5(1.0)$ & $1.5(1.1)$ & n.s. \\
\hline
\end{tabular}

* $P=0.013$ for females only

n.s. not significant

\section{Results}

Intact versus cut PCL

The results for intact and cut PCL are shown in Table 1. At the three displacement forces of 100,150 and $200 \mathrm{~N}$, the flexion gap increased from 6.8 to $9.2 \mathrm{~mm}$ when the PCL was intact and from 7.1 to $9.5 \mathrm{~mm}$ after the PCL was cut. Figure 2, which is a scatter plot of the gaps versus anterior tibial translation, demonstrates the small discrepancy of the proportions if stratified by the PCL status.

Tibial translation with both an intact and a cut PCL increased from an average of $0.6-1.5 \mathrm{~mm}$ (Table 1).

The flexion gaps differed significantly for knees with and without PCL at all three tension forces (Table 1). However, the difference between anterior tibial translation with and without PCL was not significant.
Fig. 2 Scatter plot of gaps versus anterior tibial translation stratified by the PCL status. Straight lines: regression graphs. Dashed lines: upper and lower $95 \%$ confidence intervals. Overlapping numbers are also coloured in red

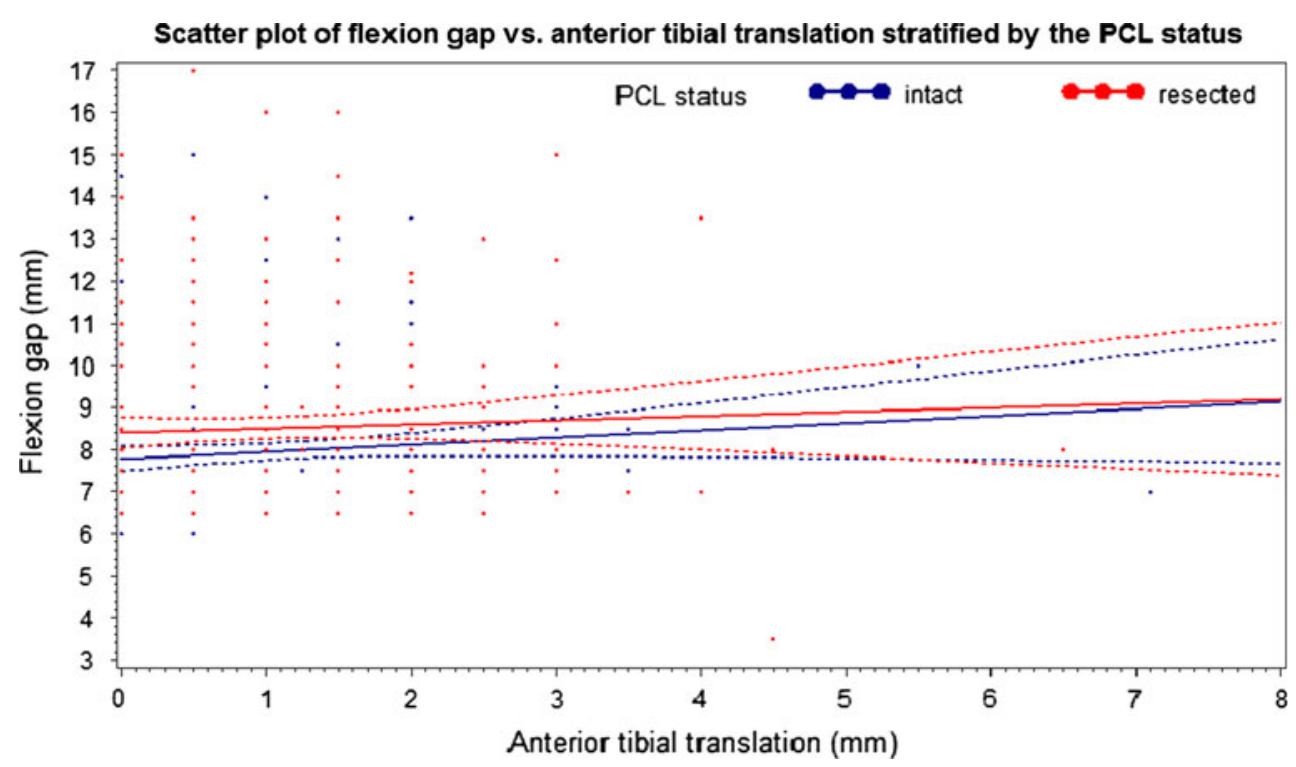


The correlation between the applied force and flexion gap when the PCL was intact or cut was high (Spearman coefficient of 0.7 and 0.6 , respectively). The correlation between the applied force and anterior tibial translation when the PCL was intact or cut was also present, though lower (Spearman coefficient of 0.5 and 0.4 , respectively).

Relationship between the gap size and anterior tibial translation for intact PCL

$\Delta$ gap1 was 0 in one knee and $\Delta$ gap2 was 0 in two knees; slope could not be calculated for these knees. The mean PCLslope1 $(n=87)$ was 0.8 (SD 1.6, 95\% CI 0.4-1.1) and the mean PCLslope2 $(n=86)$ was $0.4(\mathrm{SD} 1.4,95 \% \mathrm{CI}$ 0.1-0.7).

There was no statistically significant difference between PCLslope 1 and PCLslope2 for knees with PCL. Combining the slopes $(n=173)$, the resulting mean PCLslope was 0.6 (SD 1.4, 95\% CI 0.3-0.8). Thus, for each increase of $1 \mathrm{~mm}$ in the flexion gap, an average increase of $0.6 \mathrm{~mm}$ in anterior tibial translation occurred.

Relationship between the gap size and anterior tibial translation for resected PCL

$\Delta$ gap1 was 0 in three knees, and $\Delta$ gap2 was 0 in two knees; slope could not be calculated for these knees. The mean PCLslope $1(n=85)$ was 0.4 (SD $0.5,95 \%$ CI $0.3-0.5)$ and the mean PCLslope $2(n=86)$ was 0.5 (SD $1.3,95 \%$ CI $0.3-0.8)$.

There was no statistically significant difference between PCLslope1 and PCLslope2 for knees without PCL. Combining the slopes $(n=171)$, the mean PCLslope was 0.4 (SD 1.0, 95\% CI 0.3-0.6). Thus, for each increase of $1 \mathrm{~mm}$ in the flexion gap, a mean increase of $0.4 \mathrm{~mm}$ in anterior tibial translation occurred.

\section{Discussion}

The most important finding of the study was the fact that tibial translation with an intact and a resected PCL in less invasive total knee replacements was similar. Thus, our hypothesis suggesting pronounced tibial translation in knees with intact PCL was not confirmed. The two different situations for each knee, with and without posterior cruciate ligament, were assessed using methods from the earlier study by Christen et al. [2]. In contrast to the previous study, the patients were treated using a less invasive midvastus surgical approach and different knee prosthesis.

The size of the flexion gap in the current study differed significantly for knees with and without PCL at all different tension forces. This was expected. The PCL acts against posterior tibial translation with respect to the femur and is thought to be responsible for the anterior translation of the tibia when the flexion gap is under tension. Following cutting the PCL, the increase in the flexion gap was smaller in our study than that reported in the literature. Our measurements showed a flexion gap increase between 0.3 and $0.5 \mathrm{~mm}$ depending upon the tension applied. A difference in the gap size between intact and cut PCL has been variously reported as $2.3 \mathrm{~mm}$ by Ochsner et al. [21], 3-4 mm by Dorr et al. [7], $4.6 \mathrm{~mm}$ in medial and $4.8 \mathrm{~mm}$ in lateral approach by Kadoya et al. [15] and between 2.6 and $3.5 \mathrm{~mm}$ by Matsumoto et al. [20]. As applied tension increases, so too does the gap [2]; the differing values in these studies could be due to different forces applied to stress the gap. Furthermore, the retracting force of the extensor apparatus in the less invasive approach, during which the patella was only subluxed laterally, could result in a smaller increase in the gap.

The maximum absolute difference of $0.1 \mathrm{~mm}$ between the mean values for anterior tibial translation with and without PCL (Table 1, at $150 \mathrm{~N}$ ) was not significant. A larger sample likely would have produced a significant result. However, a maximum difference of $0.1 \mathrm{~mm}$ probably has no clinical relevance.

In contrast to our findings, Cromie et al. [3] reported roughly twice as much anterior translation after removing the PCL. It is important to note that the PCL is not the only anatomical structure that can influence tibial translation. An intact posterior capsule also must be considered, especially since it is not disrupted by anterior dislocation of the knee in less invasive TKR. Progressive, force-dependent anterior translation of the tibia therefore could depend more on the PCL in some knees, while the response of others depends more on the posterior capsule. Either can produce a more posterior femorotibial contact point in $\mathrm{CR}$ TKR when the flexion gap is too tight, or when too lax, unpredictable behaviour. Yong et al. examined factors influencing flexion gap tightness in CR TKRs. According to his results, the only significant influencing factor was tibial slope, though all knees in his study underwent a TKR via subvastus approach [11].

Summarizing the slope calculation, each increase of $1 \mathrm{~mm}$ in flexion gap means an increase in anterior tibial translation of $0.6 \mathrm{~mm}$ for intact PCL knees. This result differed from that of the previous study [2], in which a 1 -mm flexion gap increase led to a $1.25-\mathrm{mm}$ increase in anterior tibial translation. The reason for this discrepancy probably lies in different operation techniques associated with the different surgical approaches and different TKR implants. In less invasive TKRs, e.g., the extensor apparatus may influence anterior tibial translation as the laterally subluxed patella is positioned more anteriorly than in conventional approaches. Furthermore, the amount of knee 
flexion when performing the measurements could have an influence. In the previous study, the knee was put in $97^{\circ}$ of flexion for parallel alignment of the tibial and posterior femoral cuts, with $7^{\circ}$ of posterior tibial slope and $0^{\circ}$ oriented coronal femoral osteotomies (in line with femoral shaft axis) [2]. In the present study, the knee angle was $108^{\circ}$, with $3^{\circ}$ of posterior tibial slope and $15^{\circ}$ of ascending osteotomies in the posterior condyles. From a mechanical point of view, it is imaginable that the force leading to an anterior translation of the tibia due to rising tension in the PCL or the posterior capsule diminishes with greater flexion.

Thirteen patients who were not included in this study underwent TKR with a Journey implant via lateral approach including tuberosity osteotomy that was assessed using the same measurements. Although the sample size was small, an in-house analysis showed significant differences between midvastus and lateral approach in flexion gaps and tibial translations. A sufficiently large patient sample should allow published confirmation of these differences.

Heesterbeek et al. [10] have reported a mean anterior tibial translation of $1.7 \mathrm{~mm}$ per 1-mm flexion gap increase in knees with a flat intact PCL, and $2.3-\mathrm{mm}$ average translation per $1 \mathrm{~mm}$ of gap distraction in knees with a steep intact PCL. The authors implanted balanSys knees with a slope of $7^{\circ}$ and carried out the measurements in $97^{\circ}$ flexion, which makes the study comparable to that of Christen et al. [2] in which a mean relation of 1:1.25 between gap height and anterior tibial translation after flexion gap distraction with a monoblock tensioner was documented. Heesterbeek et al. [10] compared the studies and explained their higher amount of tibial translation as a result of the different tensioners that were used. With a monoblock tensioner, the most restraining structure determines the amount of translation whereas with a bicompartmental tensioner, as used in the study of Heesterbeek et al. [10], the translation may be less restrained and therefore higher.

The patients in this study were operated via a less invasive midvastus approach, receiving a Journey knee. Thus, a substantial influence of the extensor apparatus should be expected. Importantly, the measurements in the study of Heesterbeek et al. [10] were conducted 3-dimensionally using a navigation system in contrast to a possibly less accurate scale on the tensioner. Finally, the different friction coefficients of our tensioner and that in the study of Heesterbeek et al. [10] may have also contributed to the different results.

As already mentioned, in a less invasive midvastus surgical approach, the extensor apparatus could negatively influence knee balancing not only in the coronal but also in the sagittal plane by its tension being more pronounced than in a conventional surgery.
Further factors influencing the flexion gap and the tibial translation might be the weight of the leg and an improper flexion of the knee leading to a trapezoidal instead of rectangular flexion gap in the sagittal plane. We attempted to minimize their influence with the hydraulically stepless adjustable leg holder supporting the thigh at the level of the tourniquet.

The change of flexion gap with and without PCL in our study was low $(0.3-0.5 \mathrm{~mm})$ and may be adjoining the accuracy of the measuring device. Nevertheless, e.g. choosing a 2.5 -mm-thicker PE insert would mean shifting of the femorotibial contact point $1 \mathrm{~mm}$ more posteriorly due to anterior tibial translation. The influence of flexion gap was more prominent in other studies, in which CR TKR and classical invasive approach were used. This reflects difficulties in accurate balancing of flexion gap in CR TKR, which remains a difficult task [28]. Based on our clinical experience, we think that balancing the flexion gap in an appropriate way for the medial and lateral collateral ligaments, and for anterio-posterior stability is difficult in less invasive TKR using the currently available tensioning devices. This is supported by the fact that the results of our previous study were not completely reproducible in this study. This may also explain differences between the published studies $[2,7,15,21]$. Optimal balancing may be possible only with a tensioner that would be able to balance after setback of the extensor apparatus, thus simulating the closed knee situation. For a PCL-retaining TKR, the question remains also as to how one should balance the PCL, since an optimal force for gap distraction was not found yet [9].

In general, it is difficult to find arguments for preserving and balancing the PCL in CR TKR. It is well accepted that the PCL shows mild-to-severe degenerative changes in arthritic knees. In addition, an important part of the system holding and guiding the PCL, which includes the anterior and posterior menisco-femoral ligaments, is destroyed when the remnants of the menisci are cut away. Moreover, the tibial insertion of the PCL is in danger when performing tibial osteotomy. Even if an ideal PCL tension could be created, ligament function would never be the same as it was. This is also due to the fact that the ACL is missing and the PCL has to counteract the congruency of the PE insert of the prosthesis. It seems that a CR TKR functions despite these facts, and not because of an optimally balanced flexion gap.

This study was undertaken to contribute to the attempt to balance correctly an intact PCL in CR TKR and thus to create a correct femoro-tibial contact point preventing too tight or too loose flexion gap. The lack of difference in tibial translation with intact or resected PCL in the study, though, brings up more questions than answers when trying to balance correctly the PCL, because a lot of factors seem 
to interfere. For the moment, the PS TKR seems to remain the more secure way to replace an arthritic knee than a CR TKR with an uncertain behaviour of an incorrectly balanced PCL with unknown degenerative changes.

\section{Conclusion}

The results in the present study do not confirm our initial hypothesis that tibial translation is more pronounced in knees with an intact PCL than in those after PCL resection in less invasive total knee replacements. The reasons for this may include the lack of an accurate tensioner allowing simulation of a closed knee situation, and other soft tissue structures that prevent anterior tibial translation, such as the collateral ligaments, and/or the extensor apparatus. Moreover, the knee flexion angle for the used specific implant could play a role.

Open Access This article is distributed under the terms of the Creative Commons Attribution Noncommercial License which permits any noncommercial use, distribution, and reproduction in any medium, provided the original author(s) and source are credited.

\section{References}

1. Banks SA, Markovich GD, Hodge WA (1997) In vivo kinematics of cruciate-retaining and -substituting knee arthroplasties. J Arthroplasty 12(3):297-304

2. Christen B, Heesterbeek P, Wymenga A, Wehrli U (2007) Posterior cruciate ligament balancing in total knee replacement: the quantitative relationship between tightness of the flexion gap and tibial translation. J Bone Joint Surg Br 89(8):1046-1050

3. Cromie MJ, Siston RA, Giori NJ, Delp SL (2008) Posterior cruciate ligament removal contributes to abnormal knee motion during posterior stabilized total knee arthroplasty. J Orthop Res 26(11):1494-1499

4. Dejour D, Deschamps G, Garotta L, Dejour H (1999) Laxity in posterior cruciate sparing and posterior stabilized total knee prostheses. Clin Orthop Relat Res 364:182-193

5. Dennis DA, Komistek RD, Mahfouz MR (2003) In vivo fluoroscopic analysis of fixed-bearing total knee replacements. Clin Orthop Relat Res 410:114-130

6. Dennis DA, Komistek RD, Mahfouz MR, Haas BD, Stiehl JB (2003) Multicenter determination of in vivo kinematics after total knee arthroplasty. Clin Orthop Relat Res 416:37-57

7. Dorr LD, Boiardo RA (1986) Technical considerations in total knee arthroplasty. Clin Orthop Relat Res 205:5-11

8. Gollehon DL, Torzilli PA, Warren RF (1987) The role of the posterolateral and cruciate ligaments in the stability of the human knee. A biomechanical study. J Bone Joint Surg Am 69(2):233-242

9. Heesterbeek P (2010) Mind the gaps! Clinical and technical aspects of PCL-retaining total knee replacements with the balanced gap technique. Thesis, Radboud University Nijmegen Medical Center, Nijmegen. ISBN: 978-90-9025896-6

10. Heesterbeek P, Keijsers N, Jacobs W, Verdonschot N, Wymenga A (2010) Posterior cruciate ligament recruitment affects anteroposterior translation during flexion gap distraction in total knee replacement. An intraoperative study involving 50 patients. Acta Orthop 81(4):471-477
11. In Y, Kim JM, Woo YK, Choi NY, Sohn JM, Koh HS (2009) Factors affecting flexion gap tightness in cruciate-retaining total knee arthroplasty. J Arthroplasty 24(2):317-321

12. Itokazu M, Masuda K, Wada E, Ohno T, Yoshida M, Takatu T (2000) Influence of anteroposterior and mediolateral instability on range of motion after total knee arthroplasty: an ultrasonographic study. Orthopedics 23(1):49-52

13. Jacobs WC, Clement DJ, Wymenga AB (2005) Retention versus removal of the posterior cruciate ligament in total knee replacement: a systematic literature review within the Cochrane framework. Acta Orthop 76(6):757-768

14. Jacobs WC, Clement DJ, Wymenga AB (2005) Retention versus sacrifice of the posterior cruciate ligament in total knee replacement for treatment of osteoarthritis and rheumatoid arthritis. Cochrane Database Syst Rev 4:CD004803

15. Kadoya Y, Kobayashi A, Komatsu T, Nakagawa S, Yamano Y (2001) Effects of posterior cruciate ligament resection on the tibiofemoral joint gap. Clin Orthop Relat Res 391:210-217

16. Kelly MA, Clarke HD (2002) Long-term results of posterior cruciate-substituting total knee arthroplasty. Clin Orthop Relat Res 404:51-57

17. Kolisek FR, McGrath MS, Marker DR, Jessup N, Seyler TM, Mont MA, Lowry Barnes C (2009) Posterior-stabilized versus posterior cruciate ligament-retaining total knee arthroplasty. Iowa Orthop J 29:23-27

18. Mahoney OM, Noble PC, Rhoads DD, Alexander JW, Tullos HS (1994) Posterior cruciate function following total knee arthroplasty. A biomechanical study. J Arthroplasty 9(6):569-578

19. Matsuda S, Miura H, Nagamine R, Urabe K, Matsunobu T, Iwamoto Y (1999) Knee stability in posterior cruciate ligament retaining total knee arthroplasty. Clin Orthop Relat Res 366:169-173

20. Matsumoto T, Kuroda R, Kubo S, Muratsu H, Mizuno K, Kurosaka M (2009) The intra-operative joint gap in cruciateretaining compared with posterior-stabilised total knee replacement. J Bone Joint Surg Br 91(4):475-480

21. Ochsner JL Jr, Johnson WD (1994) Flexion and extension gap measurements in total knee arthroplasty after sacrifice of posterior cruciate ligament. J South Orthop Assoc 3(4):290-294

22. Pagnano MW, Hanssen AD, Lewallen DG, Stuart MJ (1998) Flexion instability after primary posterior cruciate retaining total knee arthroplasty. Clin Orthop Relat Res 356:39-46

23. Straw R, Kulkarni S, Attfield S, Wilton TJ (2003) Posterior cruciate ligament at total knee replacement. Essential, beneficial or a hindrance? J Bone Joint Surg Br 85(5):671-674

24. van Hal CT, van Hellemondt GG, Wymenga AB, Jacobs WC (2007) The anterior-posterior laxity after total knee arthroplasty inserted with a ligament tensor. Knee Surg Sports Traumatol Arthrosc 15(8):1019-1022

25. Vavrik P, Landor I, Tomaides J, Popelka S (2009) Medin modular implant for total knee arthroplasty-mid-term results. Acta Chir Orthop Traumatol Cech 76(1):30-34

26. Victor J, Banks S, Bellemans J (2005) Kinematics of posterior cruciate ligament-retaining and -substituting total knee arthroplasty: a prospective randomised outcome study. J Bone Joint Surg Br 87(5):646-655

27. Waslewski GL, Marson BM, Benjamin JB (1998) Early, incapacitating instability of posterior cruciate ligament-retaining total knee arthroplasty. J Arthroplasty 13(7):763-767

28. Wymenga AB, Christen B, Wehrli U (2005) Posterior cruciate ligament balancing in total knee arthroplasty with a dynamic PCL spacer. In: Bellemans J, Ries MD, Victor J (eds) Total knee arthroplasty: a guide to get better performance. Springer, Berlin, pp 182-187

29. Yamakado K, Kitaoka K, Yamada H, Hashiba K, Nakamura R, Tomita K (2003) Influence of stability on range of motion after cruciate-retaining TKA. Arch Orthop Trauma Surg 123(1):1-4 